\title{
A Rigorous Framework for Interactive Robot Control
}

\author{
S.STRAMigioli $\dagger$, E.D.FASSE $\ddagger$ and J.C.WILLEMS $\S$
}

This paper presents a rigorous, analytical framework for interactive control methods such as stiffness and impedance control. This paper does not present a novel synthesis method for robot control design. Rather, it presents a proper framework to analyze controllers for robots whose purpose is to interact energetically with the environment.

First geometrical tools are introduced that are used in kinematic and dynamic analysis of the spatiomechanical systems common in robotics. "Port behavior" and "behavioral deviation" are then defined both intuitively and rigorously. The utility of this framework is demonstrated by a nontrivial example. Concepts of the behavioral approach are used.

\section{Introduction}

Most tasks that robotic manipulators should be able to perform involve significant energetic interaction with the environment. Two active compliant motion control paradigms are currently distinguishable, hybrid position/force control (Raibert \& Craig 1981) and impedance control (Salisbury 1980, Hogan 1980, Hogan 1985). Many hundreds of scientific papers have been written about both topics. The physical, geometrical, mathematical foundations of hybrid control are largely in place. The basic idea is intuitive; one controls translational and rotational motion along/about some directions and controls translational forces and rotational torques along/about other directions. It was originally stated that the directions of commanded motion and of commanded wrenches were "orthogonal", assuming interaction with rigid constraints. It has been pointed out clearly in the literature (Lipkin \& Duffy 1988. Duffy 1990) that this compatibility condition is more properly expressed as one of reciprocity. Assuming interaction with frictionless constraints, commanded motions and wrenches are reciprocal and thus compatible if they produce no work.

The foundations of impedance control are not as well developed. First of all, roboticists, proponents and detractors included, do not always agree even intuitively what impedance control is! It has been said to be a kind of force control, said to be a combination of force and position control. Hogan says in his widely cited paper (Hogan 1985) that "A distinction between impedance control and the more conventional approaches to manipulator control is that the controller attempts to implement a dynamic relation between manipulator variables such as end-point position and force rather than just control these variables alone."

There is significant literature looking at the geometry of compliance and more generally impedance. Lončarić (1987) and Brockett \& Lončarić (1986) looked at the geometry of "compliance programming" using the tools of group theory. They showed that stiffness and compliance matrices could be parameterized in an intuitive way that simplified their selection. Patterson \& Lipkin (1993b, 1993a) looked at the geometry of compliance using the tools of screw theory, building upon work of Ball (1900) and Dimentberg (1965). They classify compliance in terms of screw eigenvalues and eigenvectors. Žefran \& Kumar (1997) and Howard, Žefran \& Kumar (1995) have also looked at the geometry of compliance, explaining for example differences in the structure of the stiffness matrix when defined using different implicitly defined affine connections.

Prior to defining "control of interactive behavior" of dynamic systems we call attention to two important considerations:

1. Dynamic interaction is a bidirectional physical phenomenon. The presence of an often poorly characterized environment should be considered explicitly.

2. This environment is a dynamic subsystem and not just a "signal source".

The first consideration suggests that the analysis and control of interactive systems should be performed using mathematical tools appropriate for physical systems, and not more abstract tools of mathematical systems theory. This leads to the use of dual, power-conjugate entities to describe interaction, e.g., twists and wrenches.

†University of Twente (NL), http://www.rt.el.utwente.nl/smi

$\ddagger$ Raytheon, Arizona, (USA), http: / / www . raytheon.com

$\S$ University of Groningen (NL)/ University of Leuven (B) 
The duality of twists and wrenches is nicely expressable in the framework of bond graphs (Paynter 1960). In this work a one-dimensional framework was introduced which was further developed from a thermodynamical point of view in (Breedveld 1984). Further geometrical extensions to twists and wrenches in bond graphs can be found in (Maschke, Bidard \& van der Schaft 1994) or (Stramigioli 2001). The second consideration suggests that interactive behavior control is different than standard control paradigms, which by and large consider exogenous variables to be generated by independent signal sources. In this case there is bidirectional interation between two dynamic subsystems: a robot and its environment.

Consider a robot interacting with a perhaps poorly characterized environment. So that this interation will be well behaved we want to control the robots "behavior", which is an intrinsic "something" that is independent of the environment. What is this "something"? Candidates include the so-called port or manifest variables of interaction, twists and wrenches in this case. We eliminate such variables as candidates because during interaction they depend not only on the dynamics of the robot but also on the dynamics of the environment, which constrains their values in some poorly characterized way.

The dynamic relation between twists and wrenches can be controlled independently of the environment. We therefore adopt a model-matching control paradigm (Nijmeijer \& van der Schaft 1990, Isidori 1995, Huijberts 1991), in which the robot dynamic relation emulates a desired dynamic relation. Furthermore, we formulate the model matching-problem using a behavioral framework (Willems 1991). In this way we are not forced to assume anything about the causality (input-output structure) of the robot interaction.

\subsection{Output Tracking Control and Model Matching}

Most of the problems addressed in robotics are of the output tracking form (Slotine \& Li 1991) in which a certain force or velocity reference signal should be followed as nearly as possible. We can define the problem formally as follows. Given sufficently smooth manifolds $U$ and $Y$, and given a time set $T$, let $U^{T}$ and $Y^{T}$ denote the signal spaces of $U$ and $Y$, the sets of smooth functions from $T$ to $U$ and $Y$, respectively. The time set is assumed to be a real interval.

Definition 1 Map $f: U^{T} \rightarrow Y^{T}$ is causal if given $u^{\prime}, u^{\prime \prime} \in U^{T}$ such that $u^{\prime}(t)=u^{\prime \prime}(t)$ for all $t \leq t^{\prime}$, then $f\left(u^{\prime}\right)\left(t^{\prime}\right)=f\left(u^{\prime \prime}\right)\left(t^{\prime}\right)$.

To define the robot tracking problem, assume that $U$ is a set of directly controllable joint generalized efforts or velocities. Assume that $Y$ is a set of end-effector twists and/or wrenches of interest. We assume that $Y^{T}$ is a normed space. Let $M$ be a set of measurable variables. Before stating the tracking problem it is necessary to define the set of admissible controllers for a robot.

Definition 2 Given a robot modelled by a causal mapping, $r: U^{T} \rightarrow Y^{T} \times M^{T}$, we will define the set $C$ of admissible controllers for the robot $r$, to be the set of causal mappings $c: M^{T} \rightarrow U^{T}$ for which there exist one and only one solution $(u, y, m) \in U^{T} \times Y^{T} \times M^{T}$ to the system with equations $(y, m)=r(u)$ and $u=c(m)$.

The previous definition is important because it is now possible to associate a unique $y \in Y^{T}$, corresponding to the output of the feedback controlled robot, to any $c \in C$. We will indicate the output signal $y$ corresponding to an admissible controller $c \in C$ with $y(c)$.

Definition 3 Given a robot described by a causal mapping $r$, given $C$ the set of admissible controllers for $r$, and given $y_{d} \in Y^{T}$ a desired trajectory. The output tracking problem is to find a controller $c \in C$ that minimizes $\left\|y_{d}-y(c)\right\|$.

In the tracking paradigm the goal is for output variables of interest to equal a reference signal. This paper uses the model-matching paradigm (recent references on this topic that are particularly relevant to the present paper are (Nijmeijer \& van der Schaft 1990. Isidori 1995), in which the goal is for some inputoutput relation to equal a reference input-output relation. To define this problem formally consider $Y$ to be the Cartesian product of sets $Y_{1}$ and $Y_{2}$, which can be thought as being the sets of end-effector velocities and forces. In order to proceed in this input-output framework we hypothesize that the robot can be modelled as a causal mapping of the form:

$$
f: U^{T} \times Y_{1}^{T} \rightarrow M^{T} \times Y_{2}^{T}
$$


in which $U$ and $M$ are defined as before. Intuitively this means that we consider the robot dynamics to be a mapping from the controllable inputs $U$ and the forces (respectively velocities) $Y_{1}$ of the end-effector to some measurable variables $M$ and the velocities (respectively forces) $Y_{2}$ of the end-effector. We are now ready to state the model matching problem in an input-output framework.

Definition 4 (Model-Matching Problem) Given a robot modelled by a map of the form (1.1), and a causal reference mapping $r: Y^{1} \rightarrow Y^{2}$. The model-matching problem (MMP) is to find a causal mapping (if it exists) $c: M^{T} \rightarrow U^{T}$ such that

$$
\forall y_{1} \in Y_{1}^{T}, \exists m \in M^{T} \text { s.t. } f\left(y_{1}, c(m)\right)=\left(r\left(y_{1}\right), m\right)
$$

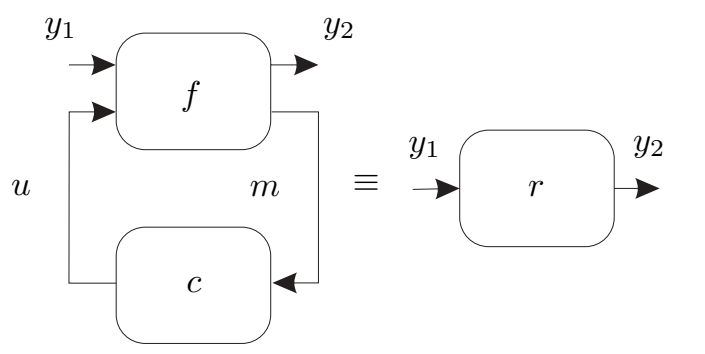

Figure 1: Representation of the I/O MMP

A $c$ solving the MMP is achievable only theoretically. Practically it is useful to have a measure of the "closeness of this achievement". A graphical representation of the definition is given in Fig. 1.

A problem with this definition is that it forces us to decompose $Y$ into two subspaces $Y_{1}$ (inputs) and $Y_{2}$ (outputs). This input/output decomposition is artificial and cumbersome and strictly related to the input output structure of the reference model. The following one-dimensional example is meant to give intuition into what is meant by interactive control. This example is derived from an example of (Driels 1996).

\subsection{Example: a Wave Energy Generator}

A wave energy generator consists of stationary and rotating assemblies. The rotor is attached to a large tank known as a duck, which is acted upon by water waves (see Fig. 2). The axis of the generator is assumed to be perpendicular to the direction of wave propogation. Relative motion of the stator and rotor drives hydraulic pumps, which is the means of energy extraction. An electric motor between the stator and rotor is available for control purposes.

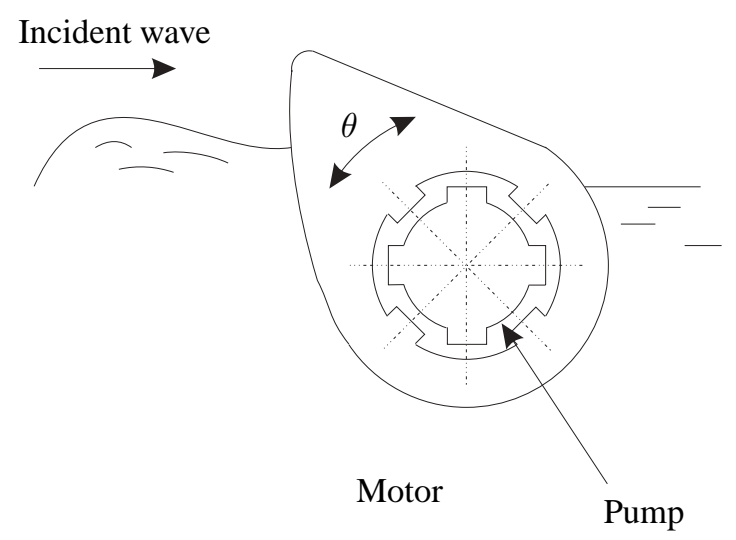

Figure 2: Wave Energy Generator

The waves have a slowly-varying period corresponding to a slowly varying, known frequency $\omega_{w}$. The purpose of this system is to generate energy. It does not make sense to say that we want to control the 
torque or orientation of the duck. It does make sense to say that we want to control the duck behavior so that it resonates at the wave frequency. This maximizes the energy transfer from the waves to the duck, and ignoring inefficiencies, the net energy generation.

\subsubsection{Model of the System}

Let $\theta$ be the relative stator-rotor angle, let $\Omega:=\dot{\theta}$ be the associated angular velocity. Suppose that the mechanical system dynamics can be modelled by the following differential equation:

$$
J_{r} \dot{\Omega}+B_{r} \Omega+K_{r} \theta=\tau+\tau_{m}
$$

$J_{r}$ is the rotational inertia of the rotor, $B_{r}$ is a coefficient of viscous damping between the stator and the rotor, $K_{r}$ is the ideal coefficient of possibly existing stiffness between the stator and the rotor, $\tau$ is the torque produced by the waves, and $\tau_{m}$ is the torque produced by the motor. The velocity-dependent torque $B_{r} \Omega$ is due to energy dissipation and to the power-take-off of the pumps.

\subsubsection{Desired behavior}

We want the system to resonate at the wave frequency, $\omega_{w}$. An appropriate desired behavior is given by the following second-order differential equation

$$
J_{r} \dot{\Omega}+B_{r} \Omega+J_{r} \omega_{w}^{2} \theta=\tau
$$

The desired behavior must contain a dissipative term so that energy can be extracted from the waves.

\subsubsection{Model-Based Controller}

Suppose we have a model of the system and corresponding parameter estimates. In particular, let $\hat{K}_{s}$ be the estimate of $K_{s}$; let $\hat{J}_{r}$ the estimate of $J_{r}$. If we consider a model based design, and ignore uncertainties in the model, then one possible, albeit simplistic control law that would achieve the desired behavior is

$$
\tau_{m}=\left(\hat{K}_{r}-\hat{J}_{r} \omega_{w}^{2}\right) \theta
$$

Without having modelled the uncertainties, this control action would achieve exactly the desired result if we suppose our model to be perfect. This is a standard approach, but the goal is not tracking or stabilisation as usual, but the achievement of a certain behavior.

\subsubsection{Achieved dynamics}

Supposing to have exact measured values, the controlled system will have the behavior corresponding to the following differential equation:

$$
J_{r} \dot{\Omega}+B_{r} \Omega+\left(K_{r}-\hat{K}_{r}+\hat{J}_{r} \omega_{w}^{2}\right) \theta-\tau=0
$$

\subsubsection{Behavior deviation}

As a measure of error of model matching consider the following quantity:

$$
\begin{aligned}
\epsilon(t) & =|| J_{r} \dot{\Omega}+J_{r} \omega_{w}^{2} \theta+B_{r} \Omega-\tau|| \\
& =\left|J_{r} \dot{\Omega}+J_{r} \omega_{w}^{2} \theta+B_{r} \Omega-\tau\right| \\
& =\left|\left(J_{r}-\hat{J}_{r}\right) \omega_{w}^{2} \theta+\left(K_{r}-\hat{K}_{r}\right) \theta\right|
\end{aligned}
$$

The error has dimensions of torque and is therefore easily interpretable. As expected, a perfect model would achieve the desired behavior exactly resulting in an error of zero. With trivial additional calculations, we could give quantitative measures of the behavioral tracking error as function of the parameter variations. Note that the ultimate control algorithm is not different than standard control algorithms. What is different is the goal of control. 


\subsubsection{Observations}

This simple example shows that there are indeed practical control problems that cannot meaningfully be posed as tracking/regulation problems. It is the goal of control, not the control law algorithm, that distinguishes a behavioral controller from a tracking controller. In the absence of wave interaction the same control law (1.5) could be used for position control, but it does not make sense in this context to say that the goal is to control the position of the duck.

The standard Model-Matching Problem is concerned with abstract systems for which geometry is irrelevant, e.g., the one-dimensional system of this example. Fortunately, similar methods can be applied to mechanical systems with complex spatial geometry like robots.

\subsection{The major behavioral concept}

To conclude the introduction of the paper and point out potential problems explain the difficulty of using position and/or force control during interaction, consider the simple one-dimensional example of Fig. 3 , A

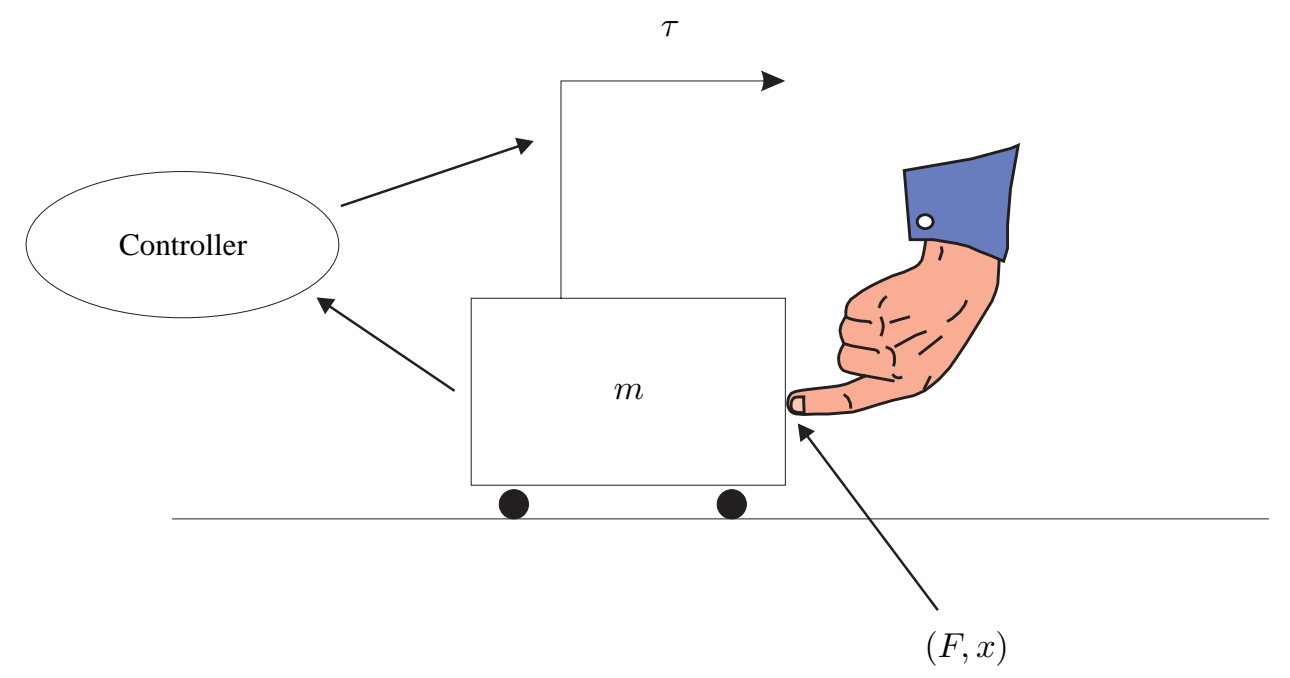

Figure 3: Representation of the I/O MMP

control force $\tau$ acts on the mass $m$ at position $x$. The environment exerts a force $F$ represented in the figure by a hand. The power exchanged between the controlled mass and the environment is therefore $P=F \dot{x}$. Using linear control, a certain robot dynamics is achieved, which can be expressed in behavioral framework (Willems 1991) in the following way:

$$
R(s)\left(\begin{array}{c}
F(s) \\
x(s)
\end{array}\right)=0, \quad R(s) \in \Re^{1 \times 2}[s]
$$

where $R(s)$ is a polynomial matrix. In the same way, the environment has its own dynamics that, assuming linearity for simplicity, can be represented in a similar way:

$$
R_{E}(s)\left(\begin{array}{c}
F(s) \\
x(s)
\end{array}\right)=0, \quad R_{E}(s) \in \Re^{1 \times 2}[s]
$$

Consider two extreme cases:

1. The mass does not contact anything: $F(t)=0 \forall t$.

2. The mass position is fixed: $\dot{x}(t)=0 \forall t$.

In the first case, the value of $x(t)$ depends only on $R(s)$, which we can directly control. In this case we can effectively use what is called Position control. In the second case, the value of $F(t)$ depends only on $R(s)$ 
which we can directly control. In this case we can effectively use what is called Force control. In all other cases both $F(s)$ and $x(s)$ depend on both the controlled robot $R(s)$ and the environment $R_{E}(s)$ :

$$
\left(\begin{array}{c}
R(s) \\
R_{E}(s)
\end{array}\right)\left(\begin{array}{c}
F(s) \\
x(s)
\end{array}\right)=\left(\begin{array}{l}
0 \\
0
\end{array}\right)
$$

The solution to the previous differential equation gives the unique solution for $F(t)$ and $x(t)$, which also depends on the imperfectly or poorly characterized environment $R_{E}(s)$.

We conclude that for interacting tasks it is questionable to control $F(t)$ and $x(t)$, which depend in part on the variable, imperfectly characterized environment. Instead one can control the only entity that is intrinsically controllable and independent of the environment: the controlled behavior $R(s)$.

\subsection{Differences with usual model matching}

From the previous section, it should be clear that for interacting mechanical subsystems it makes no sense to define a model matching problem in a causal way since the causality could change during a task execution: from position to force control or the other way around.

Furthermore, in order to assert the quality of the matching, norms are usually defined. These norms are often extra structures not justified by physical reasons and in the case the dimensions of the input/output pair is bigger than one, not even coordinate invariant (physical). In this paper we show the reason why it is NOT possible to define a physical coordinate invariant measure of the mismatch of the behavior and we will pinpoint this in a geometrical coordinate free setting.

Eventually, it should not be underestimated that multidimensional mechanical systems are complex, non-linear systems with a lot of structure, and this structure can be used to define matching for these specific systems.

\subsection{Power ports}

A major concept which will be used in the paper is the idea of a power port. A power port is the entity which describes the media by means of which subsystems can exchange physical energy with one another. A power port can be defined by the Cartesian product of a vector space $V$ and its dual $V^{*}$ :

$$
P:=V \times V^{*}
$$

Elements belonging to $P$ are pairs $(e, f) \in P$. The value of $e$ and $f$ are changing in time and shared by the two subsystems which are exchaning power through the considered port. The power exchanged at a certain time is equal to the intrinsic dual product:

$$
\text { Power }=\langle e, f\rangle
$$

This dual product is intrinsic in the sense that elements of $V^{*}$ are linear operators from $V$ to $\Re$.

\subsection{Motion of Rigid bodies, Twists and Wrenches}

In 3D mechanics, the motion, velocities and forces of rigid bodies are nicely and geometrically expressed using the concept of finite twists, instantaneous twists and wrenches respectively. The idea of the notion of a twist comes from a seminal theorem called Chasle's theorem (Stramigioli 2001). This says that any motion of a rigid body can be expressed as a rotation around a line together with a translation along the same line: a screw motion. For a finite twist, this means that we can bring any rigid body from any position in space to any other by a twist (screw motion) around and along a fixed axis in space. This axis is called the axis of the screw. The ratio between the rotation and the translation is called the pitch of the screw. For this paper, only the notion of instantaneous twists is used. In this case, we consider an instantaneous motion of a rigid body. Once again it can be seen using Chasle's theorem that we can describe any instantaneous motion as a twist around/along an instantaneous axis and having an instantaneous pitch. It can be seen (Stramigioli2001) the space of twists is equivalent in Lie group terms to the Lie algebra se(3). 
The dual of twists (linear operators on twists) are called wrenches. They correspond to the forces/moments which can be applied to a rigid body. The geometrical intuition of a Wrench is given by Poinsot's theorem (Stramigioli 2001) which dually to Chasles's theorem says that any system of forces can be expressed as a force along a line and a moment around the same line.

\subsection{Outline of Manuscript}

This paper introduces rigorously the concept of dynamic behavioral control using an acausal framework, thus eliminating the unnecessary input-output structure presented in the definition of the standard MMP. Section 2. introduces the concept of a power port. Section 3. introduces the concepts of desired and obtained behavior of a power port. Section 4 . looks at the control of behavior. Section 5. presents an application of the theory. Section 7. concludes the presentation.

\section{Energetic Variable Spaces}

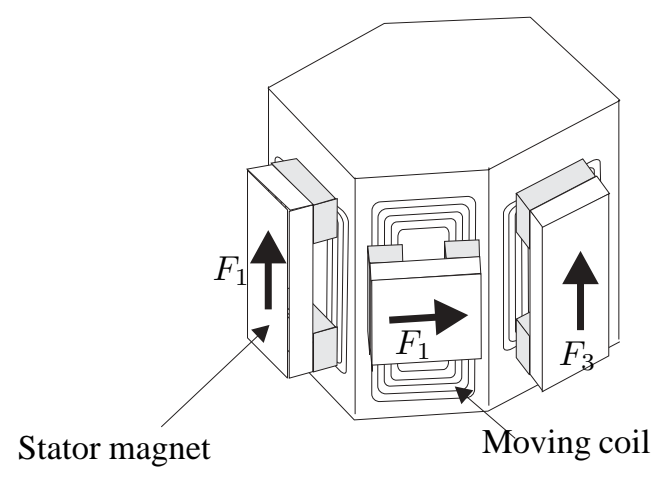

Figure 4: Haptic device example

In this and the following section, the presented concepts will be presented together with an example. The example is a haptic device (H.D.) depicted in Fig. 4, Haptic devices are an excellent example of robotic systems that interact with unstructured, poorly characterised environment: human beings. This device has been implemented and is described in (Hollis, Salcudean \& Allan 1991).

The levitated platform (flotor) is made of a lightweight, nonmagnetic material like aluminum. On this platform there are six flat-wound coils, the currents of which are controlled by servoamplifiers. Three such coils are indicated in the figure. Mounted to the stationary base (stator) of the device are six inner and outer magnet assemblies. Each assembly consists of two permanent magnets and a magnetic return plate. The inner and outer magnet assemblies are mounted in pairs forming magnetic circuits. Three of the outer magnet assemblies are indicated in the figure. Each stator magnet assembly pair interacts with a single flotor coil, exerting a Lorentz force on the coil. For small motions this force is in the plane of the coil, independent of the flotor configuration. The magnitude of this force is proportional to the coil current. By changing the six coil currents it is possible to exert arbitrary forces and moments on the flotor. A human holds the flotor where the coils are situated and can in this way interact with the device.

\subsection{Configuration Spaces}

Consider a robot consisting of a linkage of rigid bodies. Let $\mathcal{Q}$ denote the configuration space of this linkage, the set of linkage configurations. The dimension of $\mathcal{Q}$ is equal to the number of kinematic degrees of freedom of the linkage. The configuration space $\mathcal{Q}$ of H.D. is a bit different than a normal serial linkage. We can consider it to be the set of longitudinal displacements of each coil with respect to its corresponding fixed magnet. The robot interacts energetically with the environment via one or more of its links. Let $\mathcal{X}$ denote the configuration manifold (or manifold with boundary) of these links. This effector configuration manifold is often referred to as the work space. If only one link interacts with the environment we call 
this link the end-effector. For a spatial manipulator with an end-effector, the end-effector configuration manifold can be associated with the six- dimensional $S E(3)$ after a choice of reference frame. For usual robot manipulators, the space $\mathcal{Q}$ coincides with the angles and positions of the joints. If there are $n$ rigid parts of the robot which can interact with the environment like the $n$ tips of a robotic hand, one can consider $\mathcal{X}=S E(3) \times \ldots \times S E(3)$ ( $n$-times). If the dimension of $\mathcal{Q}$ is bigger than the dimension of $\mathcal{X}$, the robot is said to be kinematically redundant. The H.D. has just one end-effector with which the device can interact, namely the flotor. This implies that for the H.D. $\mathcal{X}=S E(3)$, the configuration space of the flotor.

The map from robot configurations to effector configurations, $L: \mathcal{Q} \rightarrow \mathcal{X}$, is called the direct kinematic map. This map is readily computable for serial linkages. For certain parallel linkages like the Stewart platform, the inverse map is easier to compute. The linkage is assumed to be actuated by a number of mechanical actuators, each of which has a mechanical configuration manifold, $\mathcal{A}_{i}$. The actuator configuration manifold, $\mathcal{A}=\mathcal{A}_{1} \times \mathcal{A}_{2} \times \ldots$, is the set of configurations of all of the actuators. If the dimension of $\mathcal{A}$ is bigger than the dimension of $\mathcal{Q}$, the robot is said to be redundantly actuated. The example H.D. has six actuators (coil-magnet pairs). One can consider $\mathcal{A}_{i}$ to be the relative longitudinal displacement between coil $i$ and its corresponding fixed magnet. The map from robot configurations to actuator configurations, $A: \mathcal{Q} \rightarrow \mathcal{A}$, is called the actuator kinematic map. For typical robot manipulators, $\mathcal{Q}$ and $\mathcal{A}$ can be identified, which is the case for the H.D.. In general the spaces can be very different.1

\subsection{Tangent Dynamic Spaces}

In order to study the dynamics of interaction we need to consider dynamic variable spaces associated with the port configuration manifold. Let $T_{*} \mathcal{X}$ be the tangent bundle of $\mathcal{X}$. An element $(x, v)$ of $T_{*} \mathcal{X}$ is a vector $v$ tangent to $\mathcal{X}$ at the configuration $x$. Examples of energetic tangent vectors are velocity given a configuration, $(x, v)$ and infinitesimal displacements given a configuration, $(x, d x)$. Let $T^{*} \mathcal{X}$ be the cotangent bundle of $\mathcal{X}$. An element $(x, f)$ of $T^{*} \mathcal{X}$ is a co-vector (dual vector) $f$ tangent to $\mathcal{X}$ at the configuration $x$. Examples of energetic cotangent vectors are momentum given a configuration, $(x, p)$, force given a configuration, $(x, f)$, and higher-order derivatives of momentum given a configuration, $\left(x, \frac{d^{n} p}{d t^{n}}\right)$. Tangent and cotangent vectors are not suitable for use as port variables because they are configuration-dependent; they are 'bound' vectors as opposed to 'free' vectors. In the next subsection we define free dynamic variable spaces that are suitable for use as energetic port variables.

In the example, we could have parameterized the configuration of the flotor by means of a real six-tuple $p \in \Re^{6}$ such as three Cartesian coordinates and three Euler angles. The time derivative of these variables is a numerical vector $v$ which is properly interpretable only together with the information $p$. This means that $v$ is a local velocity vector bounded to $p$.

\subsection{Free Dynamic Spaces}

Depending on what is considered to be the work space $\mathcal{X}$, one can define what shall be called free flows and free efforts. These are vector spaces independent of the configuration $x \in \mathcal{X}$. This independence is useful in the description of behaviors. Because $S E(3)$ is a Lie group, it has intrinsically associated to it a Lie algebra $s e(3)$ (the set of twists)and its dual $s e^{*}(3)$ (the set of wrenches) (Gilmore 1974). More generally the configuration manifold is $\mathcal{X}=S E(3) \times \ldots \times S E(3)$.

Definition 5 The set of free flows for a robotic system interacting with $n$ links is the following vector space:

$$
F=\underbrace{s e(3) \times \ldots \times s e(3)}_{n \text { times }}
$$

In the example, the set $F=s e(3)$ would be the set of twists of the flotor with respect to the stator. The important thing is that a twist is independent of the particular configuration of the flotor. The corresponding

\footnotetext{
${ }^{1}$ Consider the human upper limb, which has an approximately seven-dimensional configuration manifold. The actuator configuration manifold is the set of muscle configurations. The configuration manifold of each muscle is ideally a real interval $I$, so that $\mathcal{A}=I_{1} \times I_{2} \times \ldots$.
} 
diachronic space 2 is $F^{T}$, the set of diachronic (free) flows. Similarly, a free co-vector is defined to be the set of duals to $F$. Energetic covectors are often referred to as efforts.

Definition 6 The set of free efforts for a robotic system interacting with $n$ links is the following vector space dual to $F$ :

$$
E=\underbrace{s e^{*}(3) \times \ldots \times s e^{*}(3)}_{n \text { times }}
$$

In the example, $E=s e^{*}(3)$ is the set of wrenches that the human applies to the flotor. This wrench has both a torque part and a linear force part. Spaces $F$ and $E$ are geometrically and energetically dual, $F=E^{*}$. We can next define the port outcome space:

Definition 7 The port outcome space, $\tilde{W}$ is the product of the free flow and effort spaces:

$$
\tilde{W}=F \times E
$$

Port outcomes are expressily independent of configuration. To account for configuration we define an extendend port outcome space.

Definition 8 The extended port outcome space, $W$, is the set

$$
W=\mathcal{X} \times \tilde{W}
$$

The port outcome space is a vector space, the extended port outcome space is not. In this way, we have separated the configuration information from the geometrical description of the energetic variables $E$ and $F$. The corresponding diachronic spaces are $\tilde{W}^{T}$ and $W^{T}$. The duality of $E$ and $F$ makes it possible to define the instantaneous physical power of a port outcome.

Definition 9 Given $\tilde{w}=(f, e) \in \tilde{W}$ we define the instantaneous power of $\tilde{w}, \Pi(\tilde{w})$, to be the annihilation of $f$ and $e$ :

$$
\Pi(\tilde{w})=\langle e, f\rangle=\sum_{i=1}^{n}\left\langle e_{i}, f_{i}\right\rangle \quad f_{i} \in \operatorname{se}(3), e_{i} \in \operatorname{se}^{*}(3)
$$

Notice that this definition of power is independent of the configuration $x$. In the example, this power corresponds to the power that the human interacting with the H.D. supplies to the system.

\subsection{Integration of Free Vectors}

It is possible to define the temporal integral of a diachronic flow given an initial instant and corresponding initial configuration $x\left(t_{0}\right) \in \mathcal{X}$. Suppose corresponding to initial instant $t_{0} \in T$ the initial configuration is $x\left(t_{0}\right) \in \mathcal{X}$ and the initial flow is $f\left(t_{0}\right) \in F$. By means of the Lie group operation of right translation, one can associate a unique element $v \in T_{*} \mathcal{X}_{x\left(t_{0}\right)}$ to $f\left(t_{0}\right)$. We choose right translation because the free vector space is assumed to be expressed in the inertial coordinate frame. Because $v$ is a tangent velocity vector it makes sense to write $x\left(t_{0}+d t\right)=x\left(t_{0}\right)+v d t$. Integrating this differential relation we obtain the desired definition. The formal definition follows:

Definition 10 The temporal integral of diachronic flow $F(\cdot) \in F^{T}$, given time set $T \ni t_{0}$, and initial effector configuration $x_{0} \in \mathcal{X}$, denoted $\int_{T, x_{0}} F(\tau) d \tau$, is the diachronic effector configuration $x(\cdot) \in \mathcal{X}^{T}$ such that $R_{x^{-1}(t)}(x(t), \dot{x}(t))=(e, F(t))$ and $x\left(t_{0}\right)=x_{0}$, where $R_{x^{-1}}(\cdot)$ denotes the right translation of and $e$ is the identity element of the Lie group $S E(3) \times \cdots \times S E(3)$.

\footnotetext{
${ }^{2}$ Diachronic functions are variously referred to as 'signals', 'histories', 'trajectories', etc. Willems refers to them as 'signals' in his behavioral framework, but 'signal' is also commonly used to refer to a time-varying entity for which there is no significant associated power flow, such as control, measurement, and other information signals.
} 


\section{Port Behavior}

The goal of 'dynamic behavioral control' is to 'control dynamic behavior'. This implies (1) that there is some sort of 'desired dynamic behavior' corresponding to the reference model of the input-output model matching; and (2) that the mechanical system has some 'actual dynamic behavior' that a control system influences in such a way as to make it more like the desired behavior. This section defines these concepts.

The universe of port outcomes, $\mathcal{U}$, is the set of defined port outcomes given a time set under a certain constraint. Using the behavioral framework, the behavioral universe is defined to be the set of all combinations of diachronic port outcomes.

Definition 11 The universe of port outcomes is the set

$$
\mathcal{U}=\left\{(x, f, e) \in W^{T} ;\left(R_{x^{-1}}(t)\right)_{*}(x(t), \dot{x}(t))=(e, f(t)) \forall t \in T\right\} .
$$

This is indeed well defined. For each instant $t,(x(t), \dot{x}(t)) \in T_{*} \mathcal{X}_{x(t)}$ is a velocity vector tangent at $x(t)$. The Lie group map $\left(R_{x^{-}}\right)_{*}(\cdot)$ applied to a vector $(x, \cdot) \in T_{*} \mathcal{X}$, gives a vector tangent at the identity $e \in$ $\mathcal{X}$. In the example, this represents all the possible evolutions between the twist, wrench, and configuration during any interaction of a human with the H.D.. There is at least one other candidate definition for the universe of outcomes. Defining $\mathcal{U}$ to be $T^{*} \mathcal{X}$, the cotangent bundle of $\mathcal{X}$, would simplify describing behavior using Hamiltonian methods. The pair $\left(x_{0}, p_{0}\right)$ would then correspond to a reference configuration and momentum at the reference instant. The major problem with this definition is that 'momentum' is not an inherent attribute of a linkage of rigid, ideally non-inertial, bodies, and possibly redundant. Similar to (Willems 1991), a port behavior is defined to be a subset $\mathcal{B} \subset \mathcal{U}$. The port behavior defines which port outcomes are compatible.

\subsection{Port Jet Spaces}

Defining port behavior to be a subset of $\mathcal{U}$ is conceptually sufficent. For practical reasons, it is useful to be able to describe a certain behavior by means of differential equations. This can be done using jet spaces (Olver 1993). Let $\mathcal{G}$ be a manifold. Given a sufficently smooth function $g(\cdot) \in \mathcal{G}^{T}$, its $n$-th derivative with respect to $t$ is well defined. Let $G_{i}$ denote the set of all possible instantaneous $i$-th temporal derivatives for any possible $g(\cdot)$. One can then define the set $G^{(n)}=G \times G_{1} \times \ldots \times G_{n}$. Points in $G^{(n)}$ are denoted by $g^{(n)}$. Consequently, there is an induced function $g^{(n)}(\cdot)=\mathrm{pr}^{(n)} g(t)$ called the $n$-th prolongation of $g(\cdot)$, which is defined by the equation:

$$
g^{(n)}(t)=\left(g(t), g_{1}(t), . ., g_{n}(t)\right)
$$

where

$$
g_{n}(t)=\left.\frac{d^{n} g}{d t^{n}}\right|_{t}
$$

Thus $\operatorname{pr}^{(n)} g(\cdot)$ is a function from $T$ to the space $G^{(n)}$. We define a port jet space according to the theory of differential equations.

Definition 12 The $n$-th order port jet space of $T \times \tilde{W}$ is defined to be the space $T \times \tilde{W}^{(n)}$, where $\tilde{W}$ is defined in Def. 7

The port jet space is insufficient because it does not contain configuration information. Configuration information is necessary to describe static, compliant behaviors. We thus define an extended port jet space.

Definition 13 The extended $n$-th order port jet-space is defined to be the space $T \times W^{(n)}$, where $W^{(n)}=$ $\mathcal{X} \times \tilde{W}^{(n)}$.

It is now possible to associate with each point in $\mathcal{U}$ a unique element of the extended $n$-th order port jet space by means of prolongation. In general given a set $\mathcal{S}$, set $2^{\mathcal{S}}$ is the set of functions from $\mathcal{S}$ to the binary set $2=\{0,1\}$. Interpreted as membership functions, elements of $2^{\mathcal{S}}$ define subsets of $\mathcal{S}$. Thus set $2^{\mathcal{S}}$ can 
be identified with the set of subsets of $S$. Define projection onto the extended $n$-th order port jet space to be the mapping $\pi_{n}: \mathcal{U} \rightarrow 2^{T \times W^{(n)}}$ defined by the recipe

$$
\pi_{n}(x(\cdot), \tilde{w}(\cdot))=\left\{\left(t, w^{(n)}(t)\right) ; t \in T\right\}
$$

More explicitly the projection map is

$$
\begin{aligned}
\pi_{n}(x(\cdot), \tilde{w}(\cdot))=\pi_{n}(x(\cdot), f(\cdot), e(\cdot)) & = \\
& \left\{\left(t, x(t),(f(t), e(t)), \ldots,\left(\left.\frac{d^{n} f}{d t^{n}}\right|_{t},\left.\frac{d^{n} e}{d t^{n}}\right|_{t}\right)\right) ; t \in T\right\}
\end{aligned}
$$

In the H.D. example, the projection of order $n$ gives therefore at each time the configuration of the flotor, its twist, the applied wrench by the user and all their time derivatives up to order $n$.

Definition 14 Behavior $\mathcal{B} \subset \mathcal{U}$ is representable by a differential equation (RDE behavior) if there exists $a$ continuous function $\Delta_{v}^{\mathcal{B}}: T \times W^{(n)} \rightarrow \Re^{v}$ called an associated differential equation to $\mathcal{B}$ such that the subset of $T \times W^{(n)}$ defined by

$$
\mathcal{S}_{\Delta_{v}^{\mathcal{B}}}=\left\{\left(t, w^{(n)}\right) ; \Delta_{v}^{\mathcal{B}}\left(t, w^{(n)}\right)=0^{v}\right\}
$$

is equal to $\pi_{n}(\mathcal{B})$.

The definition of behavior as a subset of $\mathcal{U}$ is extremely general and there is no certainty whatsoever that one could find a compact definition that would characterise all the signals belonging to $\mathcal{B}$. A subset of all the possible $\mathcal{B}$ can be given a compact form as the kernel of a function. This subset is the one referenced in the previous definition.

Definition 15 An RDE behavior is of order $n$ if $n$ is the minimum $n \in N$ such that there exists a $\Delta_{v}^{\mathcal{B}}$ : $T \times W^{(n)} \rightarrow \Re^{v}$ satisfying the previous definition: $\mathcal{S}_{\Delta_{v}^{\mathcal{B}}}=\pi_{n}(\mathcal{B})$.

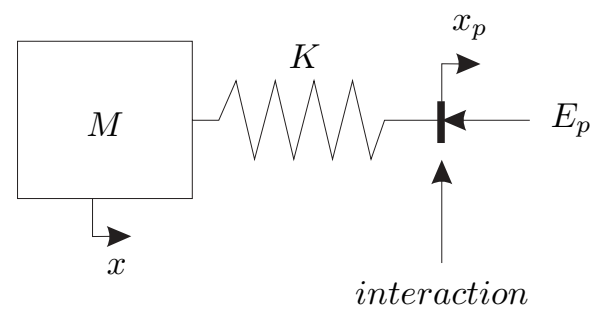

Figure 5: A simple port behavior

\subsubsection{Example}

To illustrate these last concepts, consider the system shown in Fig. 5 This is a system with trivial geometry and a work space $\mathcal{X}$ of dimension one. The equations describing the systems dynamics and interaction force are:

$$
\left\{\begin{array}{l}
M \ddot{x}+K\left(x-x_{p}\right)=0 \\
E_{p}=K\left(x-x_{p}\right)
\end{array}\right.
$$

where $x_{p}$ indicates the configuration of the port. To get a port description independent of the internal states, differentiate $E_{p}$ twice to obtain:

$$
\ddot{E}_{p}+K\left(\frac{1}{M} E_{p}+\ddot{x}_{p}\right)=0
$$

This is a scalar equation, implying that $v=1$ in Def. 14 Furthermore, the highest derivative of the port variables $(f, e)=\left(\dot{x}_{p}, E_{p}\right)$ is second order, requiring a second-order jet space description $(n=2)$. It follows that

$$
\Delta_{1}\left(t, x_{p},(f, e),\left(\frac{d f}{d t}, \frac{d e}{d t}\right)\left(\frac{d^{2} f}{d t^{2}}, \frac{d^{2} e}{d t^{2}}\right)\right)=\frac{d^{2} e}{d t^{2}}+\frac{K}{M} e+K \frac{d f}{d t}
$$




\subsection{Time-Varying and Instantaneous Behavior}

The instantaneous behavior of a robot could vary with time in order to let the robot react differently depending on the situation. This concept is related to the fact that the port behavior will in general not be time invariant. In the H.D. example, this could mean that the stiffness that the user would feel would change from one moment to another. A formal definition of what is meant with time invariant behavior can be found in Sec.8.2

Time varying behavior is useful for describing robot function. Changing the end-effector equilibrium configuration and changing principal stiffness directions are examples of behavioral changes. It is computationally useful to parametrise what is called instantaneous behavior. Let $P$ be some set of parameters. One can consider parameters varying with time within $P$. At each instant there is a time-invariant behavior determined by the instantaneous parameter. In Sec. 8.3 the differential equation structure is formally defined. It is a differential equation parameterised by the a parameter $p \in P$. If it is possible to parameterise an RDE behavior, then its parameter function at any particular time $t$ determines a time-invariant behavior. This behavior corresponds to the actual behavior only at the instant $t$, thus it will be called the instantaneous behavior at time $t$. A formal definition of this can be found in Sec. 8.4

\section{Port Behavior Control}

The previous section defined port behavior and its representation by means of differential equations. This section looks first at specifying desired behavior, using for example a differential equation structure, and then using control to make the actual behavior approximate the desired behavior. Broadly one can break the design of a behavioral controller into three steps:

1. Specify a desired port behavior.

2. Define a meaningful measure of deviation of the actual behavior from the desired behavior.

3. Specify control actions to be taken to keep the behavioral deviation within acceptable limits.

\subsection{Desired Behavior}

Desired behavior can be expressed by means of instantaneous behaviors using parametrized differential equations. Formally this means a differential equation structure $\left\{\Delta_{v}^{p}\right\}$ and its associated parameter space $P$ need to be defined. For a stiffness controller the parameters might be principal stiffness axes, principal stiffnesses, location of a center of stiffness, and an equilibrium configuration. The principal axes, principal stiffnesses and location of the center of stiffness might be piecewise constant, changing only at discrete intervals. The equilibrium configuration might move continually. This is what is done in practice. This process will be discussed in detail in the example of Sec. 5.

\subsection{Behavioral Deviation}

The importance of a measure of the deviation of the achieved behavior from the desired behavior is twofold: first it could be used as a measure in order to apply optimal control techniques for control synthesis, second it is useful for verification of the performance of a particular controller, or for comparison of the performance of different controllers.

Conceptually one could proceed as follows. Defining a metric on the space $W$, one could then use an infinity norm to define the distance between different behaviors, elements of $\mathcal{U}$. Given a measure of distance on $\mathcal{U}$, it would be straightforward to define a Hausdorff pseudometric (Abraham \& Marsden 1994) on the set of subsets of $\mathcal{U}$. This pseudometric is the intuitive metric between subsets induced by a metric between points. This would then directly give a measure of the distance between behaviors, which could theoretically be used as a measure of behavioral deviation. There are two major problems with this approach. First, given a metric on $W$, although the Hausdorff pseudometric is well defined theoretically it would be practically uncomputable and thus of little use. Second, as proven by Lončarić (1985), there does not exist an intrinsic, rigid-body-transformation-invariant metric on the set of twists, se(3). This set, 
describing both infinitesimal translations and rotations, is inhomogeneous. As such there is no intrinsic positive-definite metric or $3=S E(3) \times s e(3) \times s e^{*}(3)$, which is important for spatial manipulators. As such the resultant Hausdorff pseudometric is not intrinsically defined and thus of lesser theoretical interest. Because of these problems a more pragmatic, computable measure of deviation will be given.

Suppose the desired behavior is specified by means of a differential equation structure $\left\{\Delta_{v}^{p}\right\}$ and its associated parameter space $P$ and parameter function $p$. Let $w_{m}(t)$ denote the measured port variable at time $t$. A measure of deviation of the achieved behavior from the desired one can then be defined as follows:

Definition 16 The behavioral deviation at time $t, \epsilon(t)$, of $w_{m}(\cdot)$ from a desired RDE behavior of order $n$ represented by $\left\{\Delta_{v}^{p}\right\}$ is defined to be

$$
\epsilon(t)=\left\|\Delta_{v}^{p(t)}\left(t, \operatorname{pr}^{(n)} w_{m}(t)\right)\right\|
$$

where \|\| is the Euclidean norm on $\Re^{v}$.

The behavioral deviation is appropriately zero at instant $t$ if and only if $w_{m}(\cdot)$ satisfies the instantaneous behavior at $t$. By means of the previous definition it is also possible to determine which instantaneous behavior best describes the measured port variables. To this end consider the set of parameters minimizing the instantaneous behavioral deviation:

$$
P_{m}(t)=\arg \min _{p \in P}\left\|\Delta_{v}^{p}\left(t, \operatorname{pr}^{(n)} w_{m}(t)\right)\right\|
$$

In general $P_{m}(t)$ is a set containing multiple $p$ that minimize the behavioral deviation. In the case that $P_{m}(t)$ consists of a single $p(t)$, one could define the instantaneous behavior corresponding to $\Delta_{v}^{p(t)}$ to be the measured instantaneous behavior at time $t$.

\subsection{Behavior Control}

As illustrated in Fig. 6, a robot system can be decomposed into four major subsystems: a mechanical linkage, a set of actuators, a set of sensors, and a control system. While typical, this decomposition is somewhat artificial. For example, one can imagine sensorimotor components that are neither strictly sensors nor strictly actuators.

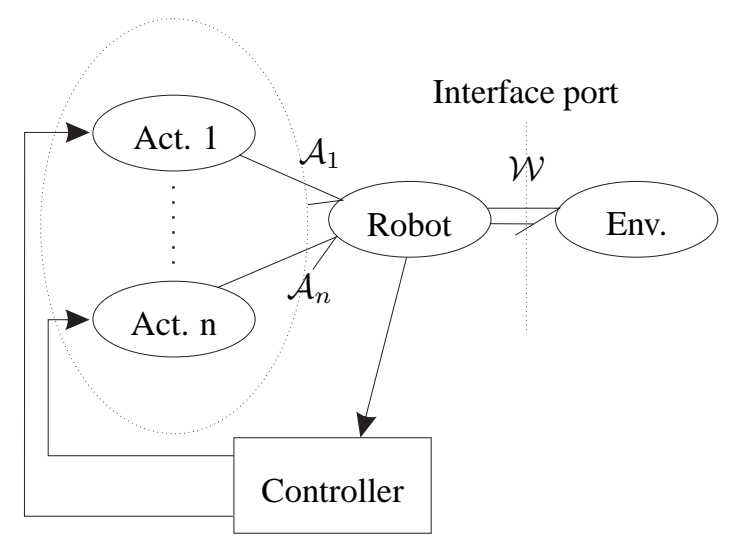

Figure 6: The Complete Controlled Schema

The energetic port of interaction with the environment is associated with the end-effector. The behavior of this port clearly depends on dynamics of the various subsystems. Figure 6 uses bond-graph notation. Half arrows indicate power bonds. Normal arrows indicate ideal signals, which do not involve significant power.

\footnotetext{
${ }^{3}$ Once a reference configuration is chosen one can identify $S E(3)$ with $\mathcal{X}$, the end-effector configuration space.
} 


\subsubsection{Robot Mechanical Linkage}

Details of the power port of the linkage end-effector have already been discussed. Besides the end-effector power port, the linkage interacts energetically with its actuators. Associated with this interaction are power ports in the space $\mathcal{A}$ (see Sec. 2.1). Usually each actuator has one degree of freedom. The total actuator configuration space is in general a subset of the Cartesian product of the individual actuators one-dimensional configuration spaces, $\mathcal{A}=\mathcal{A}_{1} \times \ldots \times \mathcal{A}_{m}$. For each actuator configuration space $\mathcal{A}_{i}$, the corresponding scalar flow (velocity) is $f_{i} \in F_{A_{i}}$; the corresponding scalar effort (force) is $e_{i} \in E_{A_{i}}$. The instantaneous power supplied by actuator $i$ to the mechanical linkage is $\Pi\left(w_{i}\right)=f_{i} e_{i}$. The individual actuator port outcome space is then $\tilde{W}_{A_{i}}=F_{A_{i}} \times E_{A_{i}}$. The corresponding extended port outcome space is then $W_{A_{i}}=\mathcal{A}_{i} \times \tilde{W}_{A_{i}}$. The universe of port outcomes for actuator $i$ is then

$$
\mathcal{U}_{A_{i}}=\left\{\left(a_{i}, f_{i}, e_{i}\right) \in\left(W_{A_{i}}\right)^{T} \text { s.t. } f_{i}=\dot{a}_{i}\right\}
$$

The universe of port outcomes for all actuators is then $\mathcal{U}_{A}=\mathcal{U}_{A_{1}} \times \ldots \times \mathcal{U}_{A_{m}}$.

The linkage external universe $\mathcal{U}_{L}^{E}=\mathcal{U} \times \mathcal{U}_{A}$ will therefore describe all the energetic ports of the mechanical linkage.

Again, let $q \in \mathcal{Q}$ denote linkage configuration (see Sec. 2.1). Let $q(\cdot) \in \mathcal{Q}^{T}$ be a joint configuration signal. Analogous to previous definitions one can define the $k$-th polongation of $q(\cdot)$ to be $q^{(k)}=\operatorname{pr}^{(k)} q(t)$ and indicate with $\mathcal{Q}^{(k)}$ the set of all possible prolongations of order $k$. The internal configuration universe can be defined as $\mathcal{U}_{\mathcal{Q}}=\mathcal{Q}^{T}$ corresponding to all the possible configuration trajectories as functions of time. We engage in an abuse of notation for $q$, considering it to be both an element of $\mathcal{Q}$ or $\mathcal{Q}^{T}$ when the meaning is obvious from context. The full linkage behaviour $\mathcal{B}_{L}$ represents all possible relations between manifest variables like the port ones of the actuators and of the workspace and the so called latent variables (Willems 1991) corresponding to the joint configuration:

$$
\mathcal{B}_{L} \subset \mathcal{U}_{L}:=\mathcal{U}_{L}^{E} \times \mathcal{U}_{\mathcal{Q}}
$$

The external (or manifest) behaviour $\mathcal{B}_{L}^{E} \subset \mathcal{U}_{L}^{E}$ can be defined as:

$$
\mathcal{B}_{L}^{E}:=\left\{\left(w, w_{A}\right) \in \mathcal{U}_{L}^{E} \mid \exists q(\cdot) \in \mathcal{U}_{Q} \text { s.t. }\left(w, w_{A}, q\right) \in \mathcal{B}_{L}\right\}
$$

For analytical purposes one can follow a procedure similar to the one in Def. 14t to define a kernel representation of the full linkage behavior by means of a differantial equation (see Sec. 8.5). Such a representation, is a function of the form

$$
\Delta_{v}^{\mathcal{B}_{L}}: T \times W^{(n)} \times W_{A}^{(p)} \times \mathcal{Q}^{(l)} \rightarrow \Re^{v}
$$

whose kernel corresponds to the behavior:

$$
\Delta_{v}^{\mathcal{B}_{L}}\left(t, w^{(n)}, w_{A}^{(p)}, q^{(l)}\right)=0^{v}
$$

where $w_{A}^{(p)} \in W_{A_{1}}^{(p)} \times \ldots \times W_{A_{m}}^{(p)}$.

It is important to realise that the linkage behavior is a design constraint. Any possible dynamical evolution of the controlled system projected on $\mathcal{U}_{L}$ will be in $\mathcal{B}_{L}$.

\subsubsection{Example of a Linkage Description}

Suppose to have an $n$ degrees of freedom ( $n$-dof) robot in which the actuators are located at the $n$ joints and therefore $\mathcal{Q}=\mathcal{A}$. By choosing a reference configuration of the end-effector it is possible to identify each end-effector configuration with an element of $S E(3)$, and thus a homogeneous matrix $H_{e}$. This is achieved by identifying any chosen end-effector configuration with the rigid body motion (element of $S E(3))$ relating the fixed reference configuration to the chosen configuration. It is then possible to express the direct kinematics as follows:

$$
L: \mathcal{Q} \rightarrow S E(3) ; q \mapsto H_{e}=\left(\begin{array}{cc}
R_{e}(q) & p_{e}(q) \\
0 & 1
\end{array}\right)
$$


where $R_{e}(q)$ is an orthonormal matrix and $p_{e}(q) \in \Re^{3}$. The derivative of this map is $D L: T_{*} \mathcal{Q} \rightarrow$ $T_{*} S E(3)$. Using the Lie group right translation of $S E(3)$, each element of $T_{*} S E(3)$ can be mapped to a twist belonging to $s e(3)$, defining the so-called geometric Jacobian (Murray, Li \& Sastry 1994):

$$
J_{a}(q): T_{q} \mathcal{Q} \rightarrow s e(3)
$$

Dually, the transpose of $J_{a}(q)$ (corresponding to the pull-back of the map) associates with each wrench $E_{a} \in s_{a}^{*}(3)$ a corresponding linkage effort $(q, \tau)=\left(q, J_{a}^{T}(q) E_{a}\right) \in T^{*} \mathcal{Q}$.

Assume that the actuator configuration space $\mathcal{A}$ can be identified with the linkage configuration space $\mathcal{Q}$. Let $\left(q, \dot{q}, \tau_{A}\right)$ denote an element of $\mathcal{U}^{A}$, the universe of actuator port outcomes. Let $\left(H_{e}, F_{a}, E_{a}\right)$ denote an element of $\mathcal{U}$, the universe of end-effector port outcomes. The linkage behavior $\Delta_{v}^{\mathcal{B}_{L}}$ can then be described using Lagrangian dyanamics and the forward kinematics as follows:

$$
\Delta_{24}^{\mathcal{B}_{L}}\left(t, w^{(0)}, w_{A}^{(0)}, q^{(2)}\right)=\left\{\begin{array}{l}
M(q) \ddot{q}+C(q, \dot{q}) \dot{q}+\tau_{f, g}(q, \dot{q})-\tau_{A}+J_{a}^{T}(q) E_{a} \\
F_{a}-J_{a}(q) \dot{q} \\
H_{e}-L(q)
\end{array}\right.
$$

where $M(q)$ is a configuration-dependent mass matrix, $C(q, \dot{q}) \dot{q}$ is a tuple of Coriolis and centripetal terms, and $\tau_{f, g}(q, \dot{q})$ is a tuple of friction and gravity terms. In this example $v=24$ because there are six scalar Lagrangian dynamic equations, six scalar differential kinematic equations, and 12 scalar, dependent kinematic equations (nine for rotation and three for translation).

\subsubsection{The Actuators}

The actuators (including necessary power amplifiers) and sensors are the interface between energetic and signal domains. Each actuator $i$ has a single-dimensional power port $W_{A_{i}}$ connected to a corresponding port of the mechanical linkage. The behavior of each actuator varies with time and is modulated by its input signals. Denote the $n_{i}$-tuple of inputs for actuator $i$ by $u_{i}=\left(u_{i 1}, u_{i 2}, \ldots, u_{i n_{i}}\right)$. The set of all possible inputs for actuator $i$ is denoted by $U_{i}$. The set of all $m$ actuators' inputs will be defined as $U=U_{i} \times \ldots \times U_{m}$. Let $U_{i}^{T}$ be the signal space for the actuator $i$ control signals $u_{i}(\cdot)$. The full $i$-actuator behavior $\mathcal{B}_{A_{i}}$, will be a subset of $W_{A_{i}}^{T} \times U_{i}^{T}$. The set of behaviors is restricted to those that can be described by means of a (generally nonlinear) function of the following form:

$$
\Delta_{v}^{\mathcal{B}_{A_{i}}}: T \times U_{i} \times W_{A_{i}}^{(n)} \rightarrow \Re^{v}
$$

This is a restriction as we do not consider all the possible dependencies between $w_{A_{i}}^{(n)}$ and $u_{i} \in U_{i}$ as a consequence of eventual dependencies of partial derivatives (Olver 1993). One can then consider the total actuator behavior $\mathcal{B}_{A}=\mathcal{B}_{A_{i}} \times \ldots \times \mathcal{B}_{A_{m}}$ for the $p$ actuators, defining:

$$
\begin{aligned}
\Delta_{k}^{\mathcal{B}_{A}}: T \times U \times W_{A}^{(j)} & \rightarrow \Re^{k} \\
\left(t, u, w_{A}^{(j)}\right) & \mapsto \Delta_{v_{1}}^{\mathcal{B}_{A_{1}}}\left(t, u_{1}, w_{A_{1}}^{\left(n_{1}\right)}\right) \times \Delta_{v_{2}}^{\mathcal{B}_{A_{2}}}\left(t, u_{2}, w_{A_{2}}^{\left(n_{2}\right)}\right) \times \ldots \times \Delta_{v_{m}}^{\mathcal{B}_{A_{m}}}\left(t, u_{p}, w_{A_{m}}^{\left(n_{m}\right)}\right)
\end{aligned}
$$

where $k:=v_{1}+v_{2}+\ldots+v_{m}, j:=\max \left\{n_{1}, n_{2}, \ldots, n_{m}\right\}$ and $W_{A}^{(j)}=W_{A_{1}}^{(j)} \times \ldots \times W_{A_{m}}^{(j)}$.

Example 1 A torque servoactuator behaves ideally as an effort source modulated by a single input, so that $u_{i}=u_{i 1}=\tau_{\text {des }}$, the desired torque. The corresponding port behavior is

$$
\Delta_{1}^{\mathcal{B}_{A_{i}}}\left(t, \tau_{\text {des }}, w_{A_{i}}^{(0)}\right)=e_{i}-\tau_{\text {des }}
$$

which is independent of $f_{i}$.

The next example refers to a so-called 'variable impedance actuator' (Fasse|1995, Fasse|1994), which is an electromagnetic motor for which the equilibrium configuration, rotational stiffness and damping can be changed independently by changing electrical currents. Briefly, it is possible to change the equilibrium 
configuration open-loop by changing the direction of of the stator magnetic field. It is possible to change the stiffness by changing the magnitude of the stator magnetic field and the magnitude of a rotor current. It is possible to change the damping by changing the magnitude of damping by changing the stator current only. Velocity-dependent torques are generated by means of rotor currents induced by motion of the rotor relative to the stator field. While this actuator has been realized in the laboratory, it has not been further developed because the range of impedances achieved open-loop is smaller than that achievable using feedback control techniques.

Example 2 A variable impedance actuator behaves ideally as a rotational, mechanical system modulated by three inputs. These inputs can be identified with the desired virtual equilibrium angle, stiffness and damping, so that $u_{i}=\left(u_{i 1}, u_{i 2}, u_{i 3}\right)=\left(\theta_{v}, K, B\right)$ The corresponding first-order port behavior is

$$
\Delta_{1}^{\mathcal{B}_{A_{i}}}\left(t, u_{i}, w_{A_{i}}^{(0)}\right)=M \frac{d f_{i}}{d t}+B f_{i}+K\left(\theta_{i}-\theta_{v}\right)-e_{i}
$$

where $\theta_{i}$ and $M$ are the actual configuration and intrinsic rotational inertia of the actuator, respectively.

Let $U=U_{1} \times \ldots \times U_{p}$ be the set of inputs for all the actuators, where $p$ is the number of actuators. For example, using torque servoactuators each element of $U$ is a $p$-tuple of desired torques.

\subsubsection{The Control Law}

In general a control law can be considered to be a causal map from measured variables and desired behavior parameters to actuator input signals:

$$
c: M^{T} \times P^{T} \rightarrow U^{T}
$$

Space $M$ is the set of measured variables and can be considered to be a subset of $W^{(n)} \times W_{A}^{(m)}$. Set $P$ is the set of potentially time-varying desired behavior parameters. If for any $t \in T$ the actuator input $u(t)$ depends only on $m(t)$ and $p(t)$ then one can write $c: M \times P \rightarrow U$. If the map does not depend on measured variables then the control is said to be open-loop. For example, the effective end-effector inertia of a redundant manipulator could be changed by reconfiguring the linkage without feedback.

Set $P$ can be considered the set of inputs of the controlled system, corresponding to parameter set $P$ in Def.20. Typical parameters are equilibrium configuration and stiffness, damping and inertia matrices.

Example 3 Let $x_{v}$ be a desired virtual equilibrium configuration of the end-effector in Cartesian coordinates using chart $\phi$. Let $K_{\phi}$ be a desired Cartesian end-effector stiffness matrix. Let $B_{\phi}$ be a desired Cartesian end-effector damping matrix. Let $L_{\phi}$ be the analytic direct kinematic function, mapping joint angles to generalized coordinates. Let $J_{\phi}$ be the corresponding analytic Jacobian, relating joint velocities and generalized velocities. Assume that joint configuration and velocity are measured, and that the robot is actuated by torque servoactuators. A suitable control law is:

$$
c: M^{T} \times P^{T} \rightarrow U^{T} ;\left((q, \dot{q}),\left(K_{\phi}, B_{\phi}, x_{v}\right)\right) \mapsto J_{\phi}^{T}(q)\left[K_{\phi}\left(x_{v}-L_{\phi}(q)\right)-B_{\phi} J_{\phi}(q) \dot{q}\right]
$$

\subsubsection{The Behavioral Control Objective}

The definition of a control law was not specific to behavioral control. Before giving a formal definition of the behavior Control Problem, let us define the set of solutions of the controlled system.

Definition 17 (Controlled Solution Set) Given (1) a robotics linkage with behavior $\mathcal{B}_{L}$ represented by a differential equation $\Delta_{v}^{\mathcal{B}_{L}}(\cdot)$ as in Sec 4.3.1 and (2) a set of actuators with behavior $\mathcal{B}_{A}$ represented by a differential equation $\Delta_{k}^{\mathcal{B}_{A}}(\cdot)$ as in Sec.4.3.3. then the set of solutions for the controller $c$ with parameters $P$ is the set $\mathcal{B}_{C}\left(c, P, \mathcal{B}_{A}, \mathcal{B}_{L}\right):=\left\{\left(w(\cdot), w_{A}(\cdot), q(\cdot)\right) \in \mathcal{U}_{L}\right.$ s.t. $\left.\Delta\left(c, P, w(\cdot), w_{A}(\cdot), q(\cdot)\right)=0^{v+k}\right\}$ where

$$
\Delta\left(c, p, w(\cdot), w_{A}(\cdot), q(\cdot)\right)=\left(\begin{array}{c}
\Delta_{v}^{\mathcal{B}_{L}}\left(t, w^{(n)}, w_{A}^{(p)}, q^{(l)}\right) \\
\Delta_{k}^{\mathcal{B}_{A}}\left(t, c\left(\left(w^{(n)}, w_{A}^{(p)}\right)(t), P\right)(t), w_{A}^{(p)}\right)
\end{array}\right)
$$


For any $\mathcal{B} \subset \mathcal{U}_{L}$ one can define its natural projection $\Pi_{\mathcal{U}}$ on $\mathcal{U}$ in the following way:

$$
\Pi_{\mathcal{U}}(\mathcal{B}):=\left\{w(\cdot) \in \mathcal{U} \mid \exists w_{A}(\cdot) \in \mathcal{U}_{A}, q(\cdot) \in \mathcal{U}_{\mathcal{Q}} \text { s.t. }\left(w(\cdot), w_{A}(\cdot), q(\cdot)\right) \in \mathcal{B}\right\}
$$

The objective of behavioral control can then be defined.

Definition 18 (Behavioral Control Problem) Given a robotics system with linkage behavior $\mathcal{B}_{L}$ and actuator behavior $\mathcal{B}_{A}$. Given a desired behavior $\mathcal{B}_{D}$ represented by a differential equation structure $\left\{\Delta_{u}^{p}\right\}$ with given bounds $a, b \in \Re^{+}$. The behavioral control problem is to find a controller $c$ such that $\forall w_{S}(\cdot) \in$ $\Pi_{\mathcal{U}}\left(\mathcal{B}_{C}\left(c, p, \mathcal{B}_{A}, \mathcal{B}_{L}\right)\right)$ the behavioral deviation $\epsilon=\| \Delta_{u}^{p}\left(\operatorname{pr}^{(n)} w_{S}(t) \|\right.$ will be bounded by $-a$ and $b$ for all time, i.e., $-a \leq \epsilon(t) \leq b$.

\section{Example: Spatial Compliance Control}

This section presents a nontrivial example to illustrate the utility of the framework for analyzing spatially complex interaction controllers. "Spatial compliance control" (Fasse \& Broenink 1997) is a Euclideangeometric method for controlling the mechanical compliance of a robotic end-effector. Unlike well known compliance control methods this method does not use generalized coordinates, so it cannot be analyzed using conventional methods. Only enough detail is given to motivate the need for a rigorous analytical framework. Publications on this topic have used both body-relative and absolute twists and wrenches.

\subsection{Desired Behavior}

The primary goal of control is to achieve a desired compliant behavior, which behavior is parametrized by spatial and nonspatial parameters. Let $H_{e}$ be a homogeneous matrix representing the configuration of the end-effector. Let $H_{v}$ be a homogeneous matrix representing a desired virtual equilibrium configuration of the manipulator. The virtual equilibrium configuration is a control parameter, not necessarily a desired configuration. It corresponds to an actual equilibrium in the absence of interaction. Let $E_{a}=\left[\begin{array}{ll}f_{a}^{T} & m_{a}^{T}\end{array}\right]^{T}$ be the wrench exerted by the end-effector on a fictitious compliant element. Let $\delta F_{a}$ be the small, finite, absolute twist taking $H_{v}$ to $H_{e}$. Mathematically this can be computed by

$$
\left[\delta F_{a}\right]=\ln \left(H_{e} H_{v}^{-1}\right)
$$

where ln denotes the matrix logarithm. The desired compliant behavior for small displacements is

$$
w_{b} \approx\left[\begin{array}{cc}
K_{t} & 0 \\
0 & K_{o}
\end{array}\right] \delta F_{a}
$$

where matrices $K_{t}$ and $K_{o}$ are symmetric stiffness matrices. Matrix $K_{t}=R_{t} \Gamma_{t} R_{t}^{T}$ is the translational stiffness matrix. Columns of $R_{t}=\left[\begin{array}{lll}e_{1 t} & e_{2 t} & e_{3 t}\end{array}\right]$ are the principal axes of translational stiffness. Matrix $\Gamma_{t}=\operatorname{diag}\left(\gamma_{1 t}, \gamma_{2 t}, \gamma_{3 t}\right)$ is a matrix of principal translational stiffnesses. A displacement along any one of the principal axes results in a linear force along the same axis.

Matrix $K_{o}=R_{o} \Gamma_{o} R_{o}^{T}$ is the rotational stiffness matrix. Columns of $R_{o}=\left[\begin{array}{lll}e_{1 o} & e_{2 o} & e_{3 o}\end{array}\right]$ are the principal axes of rotational stiffness. Matrix $\Gamma_{o}=\operatorname{diag}\left(\gamma_{1 o}, \gamma_{2 o}, \gamma_{3 o}\right)$ is a matrix of principal translational stiffnesses. A rotation about any one of the principal axes results in a moment about the same axis.

For analytical purposes it is useful to define a rotational co-stiffness matrix, $G_{o}=R_{o} \Lambda_{o} R_{o}^{T}=$ $\frac{1}{2} \operatorname{tr}\left(K_{o}\right) I-K_{o}$. Matrix $\Lambda_{o}$ is a diagonal matrix of principal rotational co-stiffnesses. It follows that $K_{o}=\operatorname{tr}\left(G_{o}\right) I-G_{o}$.

Given an antisymmetric matrix $A$ it is possible to find a unique vector v $(A) \in \Re^{3}$ such that $A$ is the cross-product matrix of $\mathrm{v}(A)$. This defines a map $v$ associating antisymmetric matrices and elements of $\Re^{3}$. A configuration-wrench map that achieves the desired stiffness is then

$$
\begin{aligned}
f_{a}-K_{t} \Delta p & =0 \\
m_{a}-\mathrm{v}\left(2 \operatorname{as}\left(R_{e} R_{v}^{T} G_{o}\right)\right) & =0
\end{aligned}
$$


where $\Delta p=p_{r}-p_{v}$. The same stiffness could be achieved in different ways. This particular configurationmoment map has a particularly simple transformation property. If $R_{e}, R_{v}$ and $R_{o}$ are all subject to a rotation then $m_{a}$ is rotated by the same amount. Because of this parameters $R_{v}$ and $R_{o}$ can be chosen independently of $\Gamma_{o}$. Similarly, parameters $p_{v}$ and $R_{t}$ can be chosen independently of $\Gamma_{t}$.

It is useful to distinguish between spatial and nonspatial parameters of compliance. Let $S$ denote the set of spatial parameters, with elements of $S$ being $s=\left(p_{v}, R_{t}, R_{o}\right)$. Let $N$ denote the set of nonspatial parameters, with elements of $N$ being $n=\left(\Gamma_{t}, \Gamma_{o}\right)$. So doing the desired behavior is given by

$$
\Delta_{6}^{s, n}\left(H_{e}, F_{a}, E_{a}\right)=\left(\begin{array}{c}
f_{a}-K_{t} \Delta p \\
m_{a}-\mathrm{v}\left(2 \operatorname{as}\left(R_{r} R_{v}^{T} G_{o}\right)\right)
\end{array}\right)=0
$$

\subsection{The Model of the system}

Let $q \in \mathcal{Q}$ indicate the generalized coordinates representing the robot configuration and $\tau$ the associated generalized forces. Suppose that the linkage is such that the actuator space $\mathcal{A}$ corresponds to the joint space $\mathcal{Q}$. The dynamics of the robot are assumed to be dominated by (1) inertia, (2) load-independent, configuration dependent joint friction, and (3) gravity. The robot is actuated by a set of variable-effort actuators and interacts energetically with its environment via the end-effector. The efforts of the actuators, $\tau_{A}$, are assumed to be the generalized forces corresponding to the generalized velocities.

This corresponds to the model given in Sec. 4.3.2. The forward kinematics are given by $H_{e}=L(q)$. Absolute twists are determined from the linkage velocities by the Jacobian, $F_{a}=J_{a}(q) \dot{q}$. The linkage behavior is given by 4.30.

\subsection{Control Law and Achieved Dynamics}

Assume that linkage configuration and velocity, $q$ and $\dot{q}$, are measured or otherwise estimated. Assume that body-relative wrenches $w_{b}$ are measured, from which absolute wrenches $w_{a}$ can be computed. The set of measured variables $M$ can then be identified with $T_{*} \mathcal{Q} \times s e^{*}(3)$. Let $\hat{\tau}_{f, g}(q, \dot{q})$ be a model-based estimate of the friction and gravity terms. A suitable control law is:

where

$$
\tau_{A}=-\hat{\tau}_{f, g}(q, \dot{q})-\tau_{c o m p}+\tau_{f f}
$$

for some scalar $\alpha \geq 1$ and

$$
\tau_{f f}=(\alpha-1) J_{a}^{T}(q) w_{a}
$$

$$
\tau_{\text {comp }}=\alpha J_{a}^{T}(q)\left(\begin{array}{c}
K_{t} \Delta p \\
\mathrm{v}\left(2 \operatorname{as}\left(R_{r} R_{v}^{T} G_{o}\right)\right)
\end{array}\right)
$$

Using this control law and the robot dynamic equation (4.30) the achieved dynamics are

$$
\left(\begin{array}{c}
f_{b} \\
m_{b}
\end{array}\right)=\frac{1}{\alpha} J_{a}^{-T}\left[M(q) \ddot{q}+C(q, \dot{q}) \dot{q}+\tau_{f, g}(q, \dot{q})-\hat{\tau}_{f, g}(q, \dot{q})\right]+\left(\begin{array}{c}
K_{t} \Delta p \\
\mathrm{v}\left(2 \operatorname{as}\left(R_{r} R_{v}^{T} G_{o}\right)\right)
\end{array}\right)
$$

\subsection{Behavioral Deviation}

The resulting behavioral deviation is

$$
\epsilon(t)=\left\|\frac{1}{\alpha} J_{a}^{-T}\left(M(q) \ddot{q}+C(q, \dot{q}) \dot{q}+\tau_{f, g}(q, \dot{q})-\hat{\tau}_{f, g}(q, \dot{q})\right)\right\|
$$

The static behavioral deviation is simply

$$
\epsilon(t)=\left\|\frac{1}{\alpha} J_{a}^{-T}\left(\tau_{f, g}(q, \dot{q})-\hat{\tau}_{f, g}(q, \dot{q})\right)\right\|
$$

This shows that a large value of $\alpha$ results in (theoretically) better behavioral tracking by reducing the effects of error in the model-based term compensating friction and gravity. 


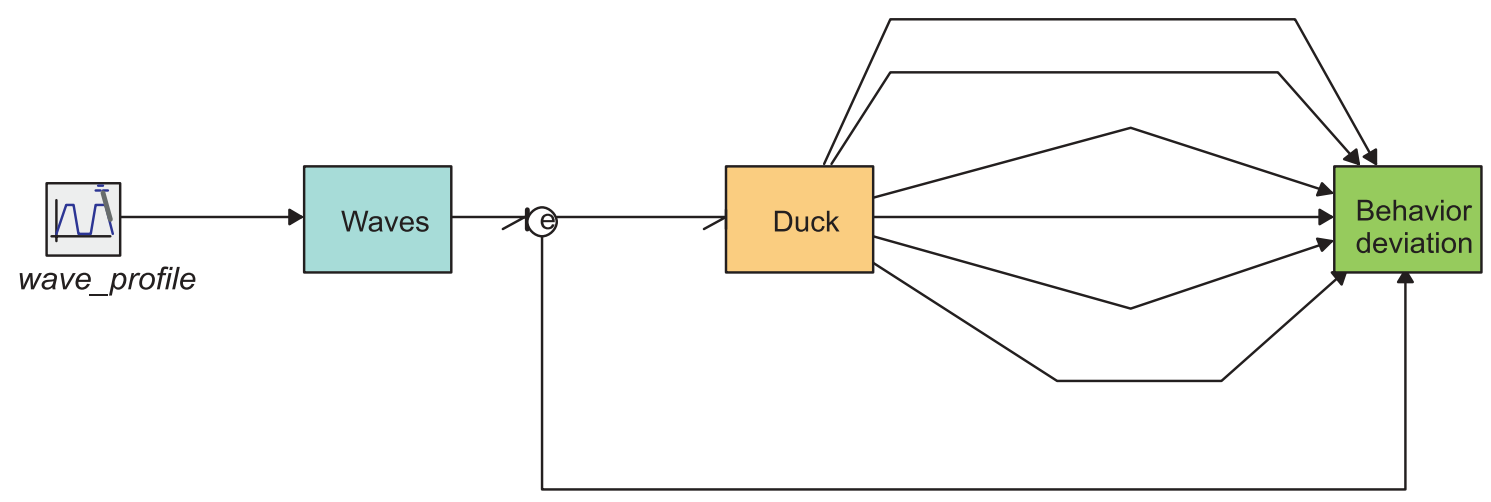

Figure 7: The main model of the Wave generator

\section{A simulation example}

To further illustrate the way in which the behavioral deviation can be used to measure the performance of a system controlled with a behavioral controller, a simple one dimensional simulation has been done which models and simulates the system reported in Fig.2 This simulation has been kept simple on purpose in order to show the basic concepts which can be easily extended to a multidimensional case.

Due to its simplicity, there is no three dimensional geometry involved. The interaction takes place through the energy exchange between the waves and the duck as explained in Sec.1.2 The chosen physical values are $J_{r}=2 \mathrm{Kg} \mathrm{m} \mathrm{m}^{2}, B_{r}=3.5 \mathrm{Nm} \mathrm{sec}$ and $K_{r}=20 \mathrm{Nm}$.

\subsection{Desired behavior}

The desired behavior for the system is reported here again for convenience:

$$
J_{r} \dot{\Omega}+B_{r} \Omega+J_{r} \omega_{w}^{2} \theta=\tau .
$$

Parameters like the resonance frequency could be estimated from an observer extracting the fundamental component (the one with the major energy content) from the wave frequency spectrum. This is nevertheless out the scope of the paper.

\subsection{The control schema}

Different control synthesis could be used, but the goal of this work is not to present new synthesis techniques, but rather analysis tools to measure the behavior deviation as presented in the paper. For matter of simplicity, the model synthesis used is a model based one which was discussed in Sec.1.2.3. The control law used to achieve the desired behavior is:

$$
\tau_{m}=\left(\hat{K}_{r}-\hat{J}_{r} \hat{\omega}_{w}^{2}\right) \theta
$$

If the controller parameters $\hat{K}_{r}, \hat{J}_{r}$ and $\hat{\omega}_{w}$ match exactly with the parameters of the duck $\left(J_{r}\right.$ and $\left.K_{r}\right)$ and with the wave frequency $\left(\omega_{w}\right)$ the desired behavior is achieved, otherwise there is a certain distance between the desired behavior and the actual one.

\subsection{The behavioral deviation}

The total system has been modeled and simulated with the package 20 sim from Control Lab Products $\mathrm{BV}$, which is able to handle port interconnection of systems. The main model is shown in Fig. 7 The block on the left generates a wave profile which exites a spring damper system (Waves). The other side of the mass-spring system is connected to the energy generator (Duck) through a power bond using the bond 

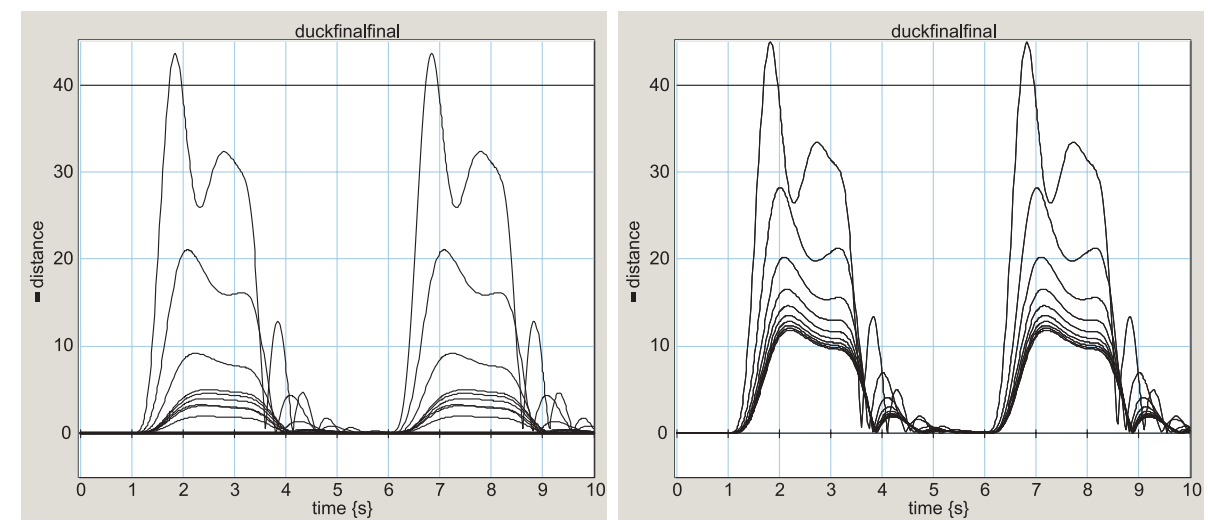

Figure 8: Period sweep with correct values of the other parameters (left) and with an error in the stiffness model value.

graph formalism. Note that indeed the arrow connecting the Waves submodel to the Duck submodel is not a normal arrow, but a 'half arrow' indicating a bi-directional signal exchange of power conjugate variables 4 . Eventually, the behavior deviation is evaluated following the line explained in Sec.1.2.5 A multiple run simulation has been performed in which a span of the period used in the model base controller is varied from 1 to 10 seconds (10 lines per plot are visible in the pictures). The result is shown in Fig.8 (left). The value corresponding to the correct wave period of $5 \mathrm{sec}$ is shown with a thick line which is to be found on the 0 distance axis. As expected, with the other parameters having an exact value, the behavior deviation is zero if the frequency is corresponding to the one of the waves. On the other hand, if the period is not well known, some deviations can be seen. Each curve on the graphic represents the deviation of the behavior of the system from the desired one, calculated by means of Eq.(1.9). As the wave period used in the control law $\left(\hat{\omega}_{w}\right)$ gets closer to the real value, the deviation gets smaller and smaller and when the period is equal to $5 s e c$ we have that the desired behavior is achieved and that the deviation is zero (the thick line on the graph.

Another simulation has been performed by choosing a wrong estimation of the model stiffness of $10 \mathrm{Nm}$ and using the same period sweep as before. The result is now shown in Fig.8 (right) where it is now possible to see, that a correct estimation of the wave period gives again the best performance corresponding to the minimum behavior deviation, but nevertheless is not equal to zero. By looking at the two plots, it can also be observed that for bigger errors of the estimated period, the difference in the overall performance changes because of the mismatch between $K_{r}$ and $\hat{K}_{r}$ which influences the behavioral mismatch and, therefore, the distance between the real behavior and the desired one.

\section{Summary}

There are two distinguishable active compliant motion control paradigms: hybrid position/force control and interactive behavior control (impedance, admittance control) (Stramigioli 1998). Currently the physical, geometrical, mathematical foundations of hybrid control are better established than those of interactive behavior control. This paper has presented an analytical framework for the behavior control of spatiomechanical robots using geometrically sophisticated control laws.

The output tracking and model matching paradigms were briefly presented. Hybrid control can be considered to be an example of the output tracking paradigm. Behavior control is related to the model matching paradigm, but the causality assumptions inherent to the model matching paradigm were considered undesirable in general. The goal of model matching control is to achieve a desired map from exogenous inputs to exogenous outputs. For interactive control there is a natural choice of exogenous variables: conjugate power variables. There will not in general be a natural choice of 'inputs' and 'outputs'.

\footnotetext{
${ }^{4}$ For details concerning the notation, the reader is addressed to standard literature of physical modeling using bond graphs.
} 
A geometrically simple, non-robotic wave energy generator example was given to motivate the idea that there are practical control problems that are not well described by the output tracking and model matching paradigms. For this system the goal of control was for the controlled duck (rotating tank) to resonate at the frequency of the incident waves. It was the goal of control, not the control law, which distinguished this controller from, say, an output tracking controller.

An essential feature of spatial robots is the nontrivial geometry of such spaces as the robot linkage configuration space and the robot end-effector configuration space. The kinematic maps relating these spaces are typically highly nonlinear. Section 2 . defined the necessary configuration spaces and tangent dynamics spaces necessary to define energetic ports. Although the presentation is unique, the material in Sec.2. is not new.

Sections 3. 5. present new material. Section 3. defined port behavior and related concepts. Initially port behavior was to defined abstractly to be a subset of the universe of port outcomes. For practical reasons we want to describe behavior more succinctly, using in particular differential equations. To this end port jet spaces and differential equation representations of behavior were defined. These concepts were illustrated using a geometrically trivial example. It is difficult to define "time-varying" and "instantaneous" behavior in a meaningful way. Definitions of these elusive concepts were proposed.

Defining behavior control is more difficult than defining, say, output tracking control. In the output tracking control paradigm the 'outputs' are assumed to be well defined and determinable at each instant in time. Intuitively one would like to say that the goal of interaction control is for the actual instantaneous behavior to track the desired instantaneous behavior as closely as possible. Section 4. defined behavioral deviation as an index of fidelity. Behavioral deviation was defined in such a way that it could be computed for any instant given a set of measured, extended port variables. The behavior control of a robot was defined, which robot consists of a mechanical linkage, a set of actuators with variable behavior, and a set of sensors. The robot behavior was defined in a way consistent with current models (e.g., using Lagrangian dynamic models of the linkage). Finally the behavioral control problem of a robot was defined.

The utility of the framework was illustrated in Sec. 5. A geometrically nontrival desired behavior was assumed. The behavior was nontrivial because of the desired, compliant orientation-torque relation. The behavioral deviation corresponding to a particular control law was computed and shown to depend strongly on one particular control parameter. This shows that the various proposed definitions were in fact defined in computable and thus useful ways. This particular, spatial controller motivated the development of the framework in the first place, because geometry cannot be ignored as it often is in discussions of generalized-coordinate-based, "Cartesian" impedance control. It is hoped that elements of the proposed framework will be useful for the development and systematic comparison of geometrically sophisticated interaction controllers of complex, spatio-mechanical robots.

\section{Appendix}

\subsection{Notation}

$\mathcal{Q}$

$\mathcal{X}$

$\mathcal{A}$

$U$

$M$

$E$

$T_{*} \mathcal{X}$

$T^{*} \mathcal{X}$

$T_{r, s} \mathcal{X}$

$T_{*} \mathcal{X}_{p}$
Configuration space

Work space

Actuator configuration space

Actuator signal input space

Measurable variable space

Euclidian Space

Tangent bundle of $\mathcal{X}$

Cotangent bundle of $\mathcal{X}$

Tensor bundle of type $(r, s)$

Tangent space of $\mathcal{X}$ at $p \in \mathcal{X}$ 


$T^{*} \mathcal{X}_{p}$
$T_{r, s} \mathcal{X}_{p}$
$C$
$\|_{C}$
$F$
$E$
$\operatorname{pr}^{(n)}$
$\tilde{W}^{(n)}$
$W$
$\mathcal{U}$
$\mathcal{B}$
$T \times \tilde{W}^{(n)}$
$T \times W^{(n)}$
$\pi_{n}$
$\Delta_{v}^{\mathcal{B}}$
$\left\{\Delta_{v}^{p}\right\}$
$\epsilon$
$A^{B}$
$[\gamma]_{x} \in T_{*} \mathcal{X}_{x}$
$0^{v}$

\subsection{Time varing behavior}

The following definition describes invariant behavior Given a port behavior $\mathcal{B} \subset \mathcal{U}$ we will say that the port behavior is time invariant if it is invariant for local group translation of the time set. A more formal definition follows. Let $(T+\tau)=\left[t_{1}+\tau, t_{2}+\tau\right]$ be the interval $T=\left[t_{1}, t_{2}\right]$ shifted by $\tau$.

Definition 19 A behavior $\mathcal{B} \subset \mathcal{U}$ is time invariant iff for any $(x(\cdot), w(\cdot)) \in \mathcal{B}$, and for any $\tau$ such that $T \cap(T+\tau) \neq \emptyset$, then

$$
\left(x^{\tau}(\cdot), w^{\tau}(\cdot)\right) \in \mathcal{B} /(T \cap(\tau+T))
$$

where $x^{\tau}(t)=x(t-\tau), w^{\tau}(t)=w(t-\tau)$, and $\mathcal{B} / T \cap(T+\tau)$ is the restriction of the behavior to the set $T \cap(T+\tau)$.

Both $x^{\tau}$ and $w^{\tau}$ are well defined on the set $T \cap(T+\tau)$. This definition is independent from any differential equation description of the behavior. For behavior representable by a differential equation the following is true.

Theorem 1 Given an $R D E$ port behavior $\mathcal{B}$ and an associated differential equation, $\Delta_{v}^{\mathcal{B}}$, then the behavior is time invariant iff $\Delta_{v}^{\mathcal{B}}\left(t_{1}, w^{(n)}\right)=\Delta_{v}^{\mathcal{B}}\left(t_{2}, w^{(n)}\right)$ for any $t_{1}, t_{2} \in T$. In this case we write $\Delta_{v}^{\mathcal{B}}: W^{(n)} \rightarrow \Re^{v}$. 


\subsection{Parameterizable behavior}

Definition 20 Let $\left\{\Delta_{v}^{p}\right\}$ be a family of differential equations indexed by parameter $p \in P$. This family is said to be a differential equation structure of an RDE port behavior $\mathcal{B}$ iff there exist a diachronic parameter $p(\cdot) \in P^{T}$ such that

$$
\Delta_{v}^{p(t)}\left(t, w^{(n)}\right)=\Delta_{v}^{\mathcal{B}}\left(t, w^{(n)}\right)
$$

where $\Delta_{v}^{\mathcal{B}}$ is a differential equation representation of $\mathcal{B}$. Set $P$ is the parameter set of the differential equation structure. Function $p(\cdot)$ is the parameter function of the behavior.

\subsection{Instantaneous port behavior}

Definition 21 Given a parametrisable RDE $n$-th order behavior $\mathcal{B}$ with parameter set $P$, parameter function $p(\cdot)$, and differential equation structure $\left\{\Delta_{v}^{p}\right\}$, then the instantaneous port behavior at time $t$ is the behavior $\mathcal{B}_{I}(t) \subset \mathcal{U}$ such that

$$
\pi_{n}\left(\mathcal{B}_{I}(t)\right)=\mathcal{S}_{\Delta_{v}^{p(t)}}
$$

where

$$
\mathcal{S}_{\Delta_{v}^{p(t)}}=\left\{\left(t^{*}, w^{(n)}\right) ; \Delta_{v}^{p(t)}\left(t^{*}, w^{(n)}\right)=0\right\}
$$

Note that in the previous definition, $t \neq t^{*}: t$ indexes the instantaneous behavior at time $t$ and $t^{*}$ indicates the time of any possible solution of the time invariant differential equation.

\subsection{Full linkage behavior}

Definition 22 We will say that the full linkage behavior $\mathcal{B}_{L}$ is representable by a differential equation (RDE behavior) if there exist integers $n, p, l$ and $v$ such that there exists a continuous function $\Delta_{v}^{\mathcal{B}_{L}}$ : $T \times W^{(n)} \times W_{A}^{(p)} \times \mathcal{Q}^{(l)} \rightarrow \Re^{v}$ such that the subset of $T \times W^{(n)} \times W_{A}^{(p)} \times \mathcal{Q}^{(l)}$ defined by:

$$
\mathcal{S}_{\Delta_{v}^{\mathcal{B}_{L}}}=\left\{\left(t, w^{(n)}, w_{A}^{(p)}, q^{(l)}\right) ; \Delta_{v}^{\mathcal{B}_{L}}\left(t, w^{(n)}, w_{A}^{(p)}, q^{(l)}\right)=0^{v}\right\}
$$

is equal to $\pi_{n, p, l}\left(\mathcal{B}_{L}\right)$, where

$$
\pi_{n, p, l}\left(w(\cdot), w_{A}(\cdot), q(\cdot)\right)=\left\{\left(t, w^{(n)}, w_{A}^{(p)}, q^{(l)}\right) ; t \in T\right\}
$$

\section{Acknowledgments}

Stefano Stramigioli would like to thank the Netherlands Organization for Scientific Research (NWO) for the support of the grant dossier number R 79-51, the Dutch Institute of System and Control (DISC) for the grant with reference TW96/DISC/022/mm. Furthermore, this work has been partially done in the context of the European sponsored project GeoPlex with reference code IST-2001-34166. Further information is available at http://www.geoplex.cc.

This research of Jan C. Willems at the University of Leuven supported by grants to SISTA from several funding agencies and sources: Research Council KUL: Concerted Research Action GOA-Mefisto 666 (Mathematical Engineering); Flemish Government: Fund for Scientific Research Flanders, project G.0256.97 (subspace); Research communities ICCoS, ANMMM, IWT (Soft4s, softsensors), Eureka-Impact (MPC-control), Eureka-FLiTE (flutter modeling); Belgian Federal Government: DWTC IUAP V-22 (20022006): Dynamical Systems and Control: Computation, Identification \& Modelling)

Cristian Secchi is thanked for his help in setting up the simulations.

\section{REFERENCES}

Abraham, R., and Mardden, J. E., 1994, Foundations of Mecahnics, ii edn, Addison Wesley. ISBN 0-8053-0102-X.

BALL, R. , 1900, A Treatise on the Theory of Screws, Cambridge at the University Press. 
Breedveld, P. , 1984, Physical Systems Theory in Terms of Bond Graphs, PhD thesis, Technische Hogeschool Twente, Enschede, The Netherlands. ISBN 90-90005999-4.

BROCKETT, R., and LONČARIĆ , J. , 1986, The geometry of compliance programming, in C. Byrnes, and A. Lindquist, eds, 'Theory and Applications of Nonlinear Control Systems', Amsterdam, North Holland, pp. 35-42.

Dimentberg, F. , 1965, The screw calculus and its applications in mechanics, Technical Report FTDHT-23-1632-67, Foreign Technology Division, Wright-Patterson Air Force Base.

DRIELS, M. , 1996, Linear Control Systems Engineering, McGraw-Hill.

DUFFY, J. , 1990, 'The fallacy of modern hybrid control theory that is based on "orthogonal complements" of twist and wrench spaces', J. of Robotic Systems 7, 139-144.

FASSE, E. D. , 1994, Taxonomy and modelling of variable impedance actuators, in 'IMACS Symposium on Mathematical Modelling', Vol. 1, Vienna, Austria, pp. 29-32.

FASSE, E. D. , 1995, Variable mechanical-impedance motors: Muscle-like, in 'Proc. of Topical Workshop of RAFT on: Control of Ambulation using FES', University of Twente, Enschede, the Netherlands, pp. 19-25.

FASSE, E. D., and BRoEninK, J. F. , 1997, 'A spatial impedance controller for robotic manipulation', IEEE Trans. on Robotics and Automation 13, 546-556.

Gilmore, R., 1974, Lie Groups, Lie Algebras, and Some of Their Applications, John Wiley \& Sons, New York. ISBN 0-471-30179-5.

Hogan, N. , 1980, Mechanical impedance control in assistive devices and manipulators, in 'Proc. Joint Automatic Controls Conf.'.

HogAn, N. , 1985, 'Impedance control: An approach to manipulation: Part I - Theory, Part II - Implementation, Part III - Applications', ASME J. of Dynamic Systems, Measurement and Control 107, 1-24.

Hollis, R., Salcudean, S., and Allan, A. , 1991, 'A six-degree-of-freedom magnetically levitated variable compliance fine motion wrist: Design, modelling and control', IEEE Trans. on Robotics and Automation .

HowArD, W., ŽEFrAn, M., and KuMAR, V., 1995, On the $6 \times 6$ stiffness matrix for three dimensional motions, in 'Proc. 9th IFToMM', pp. 1575-1579.

Huijberts, H. , 1991, Dyanamic Feedback In Nonlinear Synthesis Problems, PhD thesis, University of Twente, Enschede, The Netherlands. ISBN 90-9004142-7.

ISIDORI, A. , 1995, Nonlinear Control Systems, Communications and control engineering, iii edn, Springer Verlag, Berlin Heidelberg New York. ISBN 3-540-19916-0.

LONČARIĆ , J. , 1985, Geometrical Analysis of Compliant Mechanisms in Robotics, PhD thesis, Harvard University, Cambridge (MA).

LONČARIĆ , J. , 1987, 'Normal forms of stiffness and compliance matrices', IEEE Trans. on Robotics and Automation 3(6), 567-572.

LiPKIn, H., and DUfFY, J. , 1988, 'Hybrid twist and wrench control for a robotic manipulator', ASME J. of Mechanical Design 110, 138-144.

MaschKe, B. M., Bidard, C., and VAn Der Schaft, A. , 1994, Screw-vector bond graphs for the kinestatic and dynamic modeling of multibody systems, in 'Proc. of the 1994 ASME Int. Mechanical Engineering Congress and Exposition', Vol. DSC 55-2, Chicago, USA, pp. 637-644. 
Murray, R. M., LI, Z., and SAStry, S. , 1994, A Mathematical Introduction to Robotic Manipulation, CRC Press. ISBN 0-8493-7981-4.

NiJMeIJer, H., and VAN DER SchAFt, A. , 1990, Nonlinear Dynamical Control Systems, i edn, Springer Verlag, New York inc. ISBN 0-387-97234-X.

Olver, P. J. , 1993, Applications of Lie Groups to Differential Equations, Vol. 107 of Graduate texts in mathematics, ii edn, Springer Verlag, New-York. ISBN 0-387-94007-3.

PATterson, T., and LiPkin, H. , 1993a, ‘A classification of robot compliance', ASME J. of Mechanical Design 115, 581-584.

PATterson, T., and LiPKin, H. , 1993b, 'Structure of robot compliance', ASME J. of Mechanical Design 115, 576-580.

Paynter, H. M. , 1960, Analysis and Design of Engineering Systems, M.I.T. Press, Cambridge, Massachusetts. Course 2.751 .

RAIBERT, M., and CRAIG, J. , 1981, 'Hybrid position/force control of manipulators', ASME J. of Dynamic Systems, Measurement and Control 102, 126-133.

SAlisbury, J. , 1980, Active stiffness control of a manipulator in Cartesian coordinates, in 'Proc. IEEE Conf. on Decision and Control', pp. 95-100.

Slotine, J.-J. E., and LI, W. , 1991, Applied Nonlinear Control, Prentice Hall. ISBN 0-13-040890.

Stramigioli, S. , 1998, From differentiable manifolds to interactive robot control, PhD thesis, Delft University of Technology, Delft, The Netherlands. ISBN 90-9011974-4, http://www.rt.el.utwente.nl/smi/download/phd.pdf.

Stramigioli, S. , 2001, Modeling and IPC Control of Interactive Mechanical Systems: a coordinate free approach, LNCIS, Springer, London.

Willems, J. C. , 1991, 'Paradigms and puzzles in the theory of dynamical systems', IEEE Trans. on Automatic Control 36(3), 259-294.

ŽEFrAn, M., and KUMAR, V. , 1997, Affine connections for the Cartesian stiffness matrix, in 'Proc. IEEE ICRA', pp. 1376-1381. 